\title{
Research on Maintenance Strategy Evaluation Method for Power Equipment through Experimental Verification
}

\author{
Li Guanglei ${ }^{1, a}$, Wang Chunlin ${ }^{2, b *}$, Wu Tao ${ }^{3, c}$, Ma Mengchao ${ }^{4, d}$ and Guo \\ Deqiang ${ }^{5, e}$ \\ ${ }^{1}$ State Grid Shandong Electric Power Research Institute, Jinan, China \\ ${ }^{2}$ State Grid Linyi Power Supply Company, Linyi, China \\ ${ }^{3}$ State Grid Jibei Electric Power Research Institute Co.Ltd, Beijing, China \\ ${ }^{4}$ State Grid of China Technology College, Jinan, China \\ ${ }^{5}$ Shandong Luneng Software Technology Co. Ltd, Jinan, China \\ a lovexjtulgl@126.com, b214672717@qq.com, c qiaxw@sina.com, d sqwet@sohu.com, \\ egdq2@163.com
}

\begin{abstract}
Keywords: system risk; power transmission and transformation equipment; maintenance plan.
Abstract. A maintenance schedule evaluation method for transmission and transformation equipment considering operation risk of power system is presented. The Sequential Monte Carlo simulation is used to solve probability problems. Considering the effects of equipment maintenance, a liner programming model is made for each feasible system state randomly generated and solved by primal-dual method with infeasible initial points. The power system of Zibo is used as an example to verify the validity of the model and arithmetic presented in the paper.
\end{abstract}

\section{Introduction}

During operation in power grid, maintenance costs of transmission and transformation equipment play a significant role, so it's important to improve the efficiency of condition-based maintenance. Condition-based maintenance relies on equipment performance and the suitable opportunity [1-4]. Compared with planned maintenance, condition-based maintenance can effectively reduce equipment maintenance costs, prolong service life of equipment and improve equipment reliability. In power grid, technologies of condition-based maintenance have been obtaining more and more attention and still changing [5-10].

These benefits from proven technique of equipment condition monitoring, fault diagnosis and prediction [11]. The paper [9] discusses gradual results using real-time condition-based maintenance system of power transmission and transformation equipment using in Zhejiang provincial power company from several aspects, such as maintenance workload, operational tasks, the reliability of transmission equipment, repetition rate of transmission equipment, overall achievements of maintenance. It is obvious that the rational mode of condition-based maintenance has a strong superiority.

\section{Overall design}

Sequential Monte Carlo simulation within a certain period, power system reliability evaluation is the basis of research on maintenance schedule evaluation method strategic for transmission and transformation equipment considering operation risk of power system. In view of time correlation of maintenance programs, Sequential Monte Carlo Simulation is used as the tool for this basis.

Definitions of economic, security and environment indicators Definitions of economic, security and environment indicators are concretely expressed as follows: 
(1) Economic indicators:

$R_{1}=C+E E N S \cdot C_{k W h}+\sum_{i=1}^{n} E_{C 1 i} P_{i}$

In the formula (1), $C$ represents budgetary expense of a maintenance scheme; EENS represents Expected Energy Not Served. $C_{k W h}$ Represents cost of unit load; $C_{c l i}$ represents the mathematical expectation of unit cost of equipment maintenance; $P_{i}$ represents the cumulative probability of failure of device $i$; $n$ represents the total number of grid components. This article based on the method of the gross national product calculates unit cost of missing load.

(2) Environment indicators

$R_{2}=\sum_{i=1}^{n} E_{C 2 i} P_{i}$

In the formula (2), $E_{C 2 i}$ represents mathematical expectation of impact on the environment affected by the damage of equipment $i$; $P_{i}$ represents cumulative failure probability of equipment $i$; $n$ represents the total number of grid components.

Table1 recommended range of

\begin{tabular}{|c|c|c|c|}
\hline & slight pollution & moderate pollution & heavy pollution \\
\hline$E_{C 2 i}$ & 3 & 6 & 9 \\
\hline
\end{tabular}

(3) Safety indicators:

$R_{3}=\sum_{i=1}^{m} E E N S_{i} \cdot C_{k W h i}$

In the formula (3), EENS represents the expectations of lack of power supply substation $i$; $C_{k W h i}$ represents impact factors of safety caused by unit load loss of substation $i$; $m$ represents the total number of substations in the system.

Reference to "State Grid Corporation of power transmission equipment risk assessment guidelines", the impact on security caused by fault must consider both personal and grid, respectively accounted for $50 \%$ of the weight. Impact factors of a variety of load loss on security are shown in following table 2 , reference to the recommended range in above guidelines.

Table2 safety indicators

\begin{tabular}{|l|c|c|c|c|}
\hline \multirow{3}{*}{ load type } & \multicolumn{4}{|c|}{ safety factors } \\
\cline { 2 - 5 } & \multicolumn{2}{|c|}{ personal } & \multicolumn{2}{c|}{ grid } \\
\cline { 2 - 5 } & loss & $C_{k W h i 1}$ & loss & $C_{k W h i 2}$ \\
\hline first-class load & General & 7 & General & 4 \\
\hline second-class load & Major & 9 & Major & 7 \\
\hline third-class load & Large & 10 & Large & 10 \\
\hline
\end{tabular}

For each substation, according to the proportion of various types of load, $C_{k W h i}$ is calculated by the following formula (4).

$$
C_{k W h i}=\sum_{i=1}^{3} 0.5 \cdot\left(C_{k W h i 1}+C_{k W h i 2}\right) \cdot W_{i}
$$

In the formula (4), $i$ represents load type; 1- first-class load, 2- second-class load, 3third-class load; $W_{i}$ represents the proportion of various types of load of substation $i$.

Maintenance schedule evaluation and ordering Maintenance schedule evaluation needs to integrate its economic, environmental and security implications. Therefore, weighted sums of economic, environmental and safety losses are used in this article to evaluate the integrated consequences, calculated based on the following formula (5).

$$
R=W_{R 1} R_{1}+W_{R 2} R_{2}+W_{R 3} R_{3}
$$


In the formula (5), $R$ represents comprehensive risk of a maintenance schedule; $W_{R 1}$ 、 $W_{R 2}$ and $W_{R 3}$ represent weight coefficient of economic, security and environmental factors, respectively valued for the 0.4,0.2,0.4 according to "State Grid Corporation of transmission and distribution equipment risk assessment guidelines". $R_{1}, R_{2}$ and $R_{3}$ respectively mean economic risk, security risk and environmental risk.

\section{Analyses example}

The power system of an area power is used as an example to verify the validity of the model and arithmetic presented in this paper. The power system contains sixty-five $110 \mathrm{kV}$ and above transformer substations and 114 main transformers, which contains sixteen $220 \mathrm{kV}$ transformer substations, twenty-seven main transformers capacity of 4320MVA; forty-nine 220kV transformer substations ,87 main transformers capacity of 3799MVA. System includes $123110 \mathrm{kV}$ and above transmission lines, composed of forty-nine $220 \mathrm{kV}$ transmission line length of $825.1 \mathrm{~km}$ and seventy -four110 kV transmission line length of $763.1 \mathrm{~km}$. Electricity sales in 2007 are 17.62 billion kilowatts and maximum network load for this area is 2710MW.

Reliability evaluation Table 3 shows the results of reliability evaluation. Power Supply Reliability of Zibo Area Power System is $99.989 \%$, which is higher than the power supply reliability above 99.90\% in urban areas required by State Grid Corporation.

Table 3 reliability evaluation of Zibo Area Power System

\begin{tabular}{|c|c|}
\hline Evaluation Indexes & Results of reliability evaluation \\
\hline EENS (MW) & 1935 \\
\hline Economic Indicators (million) & 1674 \\
\hline Safety indicators (1) & 12481 \\
\hline Environmental indicators (1) & 950 \\
\hline
\end{tabular}

Maintenance schedule evaluation In this paper, three kinds of maintenance schemes are calculated and compared. Maintenance scheme 1 is an actual maintenance scheme of Zibo Power Supply Company in 2009 involving $110 \mathrm{kV}$ and above transmission and transformation primary equipment. This scheme includes projects such as maintenance, technical innovation and municipal transformation, involves 74 maintenance devices and totally invests 41.18 million. Maintenance scheme 2 is part of the equipment maintenance program, involves 45 maintenance devices and the total cost is 7.85 million; Maintenance scheme 3 is also part of the equipment maintenance program, involves 14 maintenance devices and the total cost is 36.77 million.

Table 4 maintenance schedule evaluation

\begin{tabular}{|c|c|c|c|}
\hline Type & Maintenance scheme 1 & Maintenance scheme 2 & Maintenance scheme 3 \\
\hline EENS(MW) & 1760 & 1894 & 2268 \\
\hline maintenance costs(million) & 41.18 & 7.85 & 36.77 \\
\hline economic indicators(million) & 56.33 & 23.81 & 53.34 \\
\hline safety indicators(1) & 11356 & 12221 & 14631 \\
\hline environmental indicators(1) & 835 & 887 & 3 \\
\hline comprehensive sequencing & 2 & 1 & 319 \\
\hline
\end{tabular}

Comparing Table 3 and Table 4, it can be seen that Maintenance scheme 1 and Maintenance scheme 2 reduce the total risk of the whole system in one year. The main reason for this phenomenon is due to equipment maintenance, which reduces the failure rate, improves system reliability, and decreases operational risks. The degree of operating risk reduction is greater than increased systemic risk caused by outage of maintenance equipment. Mathematical expectation of total annual load-shedding in Maintenance scheme 3, mathematical expectation of economic losses caused by total annual load-shedding, mathematical expectation of security loss caused by total annual load-shedding are all greater than corresponding value of system reliability analysis without 
maintenance plans. The main reason for this phenomenon is that the degree of system reliability improvement is less than increased systemic risk after completion of equipment maintenance.

\section{Summary}

From the perspective of the risk of power system operation, this article considers the influence of maintenance on power transmission and transformation equipment operation and evaluates maintenance schemes from the view of Economic, security and environment. Sequential Monte Carlo Simulation achieves the large-scale system reliability calculation and risk assessment, reliably simulates and analyzes the probable different phenomena in power system operation and synthesizes probability of its occurrence and consequences, thus quantitatively get risk indicators. Practice proves that this method can meet the needs of engineering calculations.

\section{References}

[1] Howard W. Penrose, Simple time-to-failure estimation techniques for reliability and maintenance of equipment, IEEE Electrical Insulation Magazine. Vol. 20, No. 7, 2009, pp.14-18.

[2] M. Marseguerra, E. Zio, and L. Podofillini, Condition-based maintenance optimization by means of genetic algorithm and Monte Carlo simulation, Reliability Engineering and System Safrty, vol. 77, no. 2,2002,pp.151-166.

[3] A. Grall, C. Berenguer, L. Dieulle, A condition-based maintenance policy for stochastically deteriorating systems, Reliability Engineering and System Safety,2002,pp.167-180.

[4] Dongyan Chen, Kishor S. Trivedi, Optimization for condition-based maintenance with semi-Markov decision process, Reliability Engineering and System Safety ,2005,pp.25-29.

[5] Chen sanyun,Tan hongen,Jiang zhigang, Condition-based maintenance for power transmission and transformation equipment, Beijing:China Electric Power Publishing House, 2004.

[6] An L ing,Jiang Xiuchen,Zhu Yu,Han Zhendong,Chen Yazhu, Object-oriented design and implementation of the state maintenance software system for transmission lines,Automation of Electric Power Systems, Apr.10, 2002,pp. 66-69.

[7] Yuan Zhijian,Sun Caixin,Li Jian,Du Lin,Liao Ruijin, Study on condition-based maintenance policy of transformer based on fuzzy multiple expert and multiple attribute group decision making,Automation of Electric Power Systems, 2004,pp.66-70.

[8] Zhang huai-yu,Zhu song-lin,Zhang yang,Lou qi-min,Zhang liang,Research and Implementation of Condition-Based Maintenance Technology System for Power Transmission and Distribution Equipment, Power System Technology, vol. 33, 2009,pp.70-73.

[9] Chen an-wei, Application of condition-based maintenance for power transmission and transformation equipmen, Power System Technology, vol. 33, 2009,pp.215-218.

[10] Lin song,Wang qing-hong,Liu ran,Condition Based Maintenance Technology of Digitized Substationt,Power System Technology, Vol. 31,2007,pp. 137-140.

[11] Jiao Shangbin,Liu Ding,Zheng Gang,Li Qi,On-line insulator contamination monitoring system for transmission lines based on telemetry, Automation of Electric Power Systems, vol. 28,2004,pp.71-75. 